\title{
Resolution of night terrors after discontinuation of cetirizine
}

\author{
Lourdes DelRosso", Romy Hoque
}

Department of Neurology, Division of Sleep Medicine, Louisiana State University School of Medicine, Shreveport, USA;

\#Corresponding Author: lordydel@yahoo.com

Received 15 July 2012; revised 22 August 2012; accepted 10 September 2012

\begin{abstract}
We present a 4-year-old girl with allergies and asthma who developed night terrors after initiation of montelukast at 2 years of age. Montelukast was discontinued and cetirizine was started. Nigh terrors persisted. Sleep diaries were collected and diagnosticpolysomnogram (PSG) was ordered. Sleep diaries revealed an average sleep time of 10 hours with night terrors occurring three nights a week approximately two hours after sleep onset. The PSG did not show evidence of sleep disordered breathing or periodic leg movements. Cetirizine was discontinued and the night terrors ceased. Upon re-introduction of the medication, the sleep terrors recurred.
\end{abstract}

Keywords: Night Terrors; Montelukast; Cetirizine; Allergic Rhinitis

\section{INTRODUCTION}

Night terrors are common in pediatric populations with a prevalence of $14.7 \%$ in children 3 - 10 years of age [1]. Night terrors usually arise from slow wave sleep (N3); start with a loud scream; are accompanied by autonomic nervous system manifestations; and have been associated with stress, gastro-esophageal reflux (GERD) and sleep disordered breathing. We present a case of night terrors in a pediatric patient after initiation of the commonly used allergy medications montelukast and cetirizine while already on beclamethasone. Cetirizine is a second-generation antihistamine that crosses the blood brain barrier. Montelukast is a leukotriene receptor antagonist used for asthma and seasonal allergies. Beclamethasone is a corticosteroid used as a nasal spray for allergic rhinitis, and used as an inhaler for asthma. The

\footnotetext{
*The authors declare that they do not have any conflict of interest. The parents of the patient signed a consent for publication of this case report.
}

night terrors resolved after discontinuation of montelukast and cetirizine.

\section{CASE REPORT}

A 4-year-old girl presents for evaluation of nocturnal episodes consisting of abrupt awakening with screaming and confusion lasting fifteen minutes in duration and occurring three nights a week. The child usually returns to sleep without difficulty following each event. She denies dream recall or memory of the event upon awakening in the morning. The events began at 2 years of age after initiation of montelukast for seasonal allergic rhinitis with nasal congestion and occasional snoring. The montelukast was discontinued and cetirizine $2.5 \mathrm{mg}$ a day was initiated. The patient's only other medications were beclomethasone $40 \mathrm{mg}$ two puffs daily, and albuterol $50 \mathrm{mcg}$ inhaler as needed for asthma.

The patient's asthma started at 1 year of age, is well controlled with the beclamethsaone and albuterol. No nocturnal asthma exacerbations/symptoms were reported. Other past medical history includes bilateral otitis media and GERD. Surgical history includes tonsillectomy and adenoidectomy at 2 years of age with subsequent improvement in snoring.

Family history is negative for parasomnias. Review of systems was positive for: snoring, nasal congestion and post-nasal drip; and negative for: behavioral disturbances, excessive daytime sleepiness, sleepwalking, sleep talking, stereotypic movements or restless legs.

Physical examination revealed a child in no distress with a body mass index of 18 . Nasal mucosa waserythematous with boggy turbinates. Oral airway exam revealed a Mallampati score of IV, with no tonsils noted. The remainder of her physical and neurological examination was unremarkable. Radioallergosorbent test (RAST) testing was negative for common animal, grass and weed antigens.

Diagnostic polysomnogram (PSG) revealed a sleep latency of 47 minutes; sleep efficiency of $89 \%$ and arousal index of 7. Apnea-hypopnea index (AHI) was 0.4. The 
minimum oxygen desaturation was $96 \%$. There was no evidence of snoring, hypoventilation, or abnormal nocturnal behaviors. Sleep diaries revealed an average nocturnal sleep time of 10 hours with no daytime napping. Night terrors were noted between midnight and two am.

Cetirizine was stopped and night terrors ceased. After three weeks, cetirizine was re-introduced with recurrence of sleep terrors.

\section{DISCUSSION}

The International Classification of Sleep Disorderssecond edition, defines night terrors as sudden episodes of terror during sleep, usually initiated by a cry or loud scream, accompanied by autonomic nervous system and behavioral manifestations of intense fear. The diagnostic criteria must include at least one of the following: difficulty in arousing the patient, mental confusion when awakened from the episode, amnesia for the episode, and potentially dangerous behaviors. The condition must not be related to another sleep disorder; medical, mental or neurological condition; medication or substance use [2].

Sleep terrors are a common parasomnia, sometimes confused with nightmares. Genetic factors, sleep deprivation, stress and fever play an important role in their manifestation [3,4]. Sleep terrors occur in the first couple of hours of sleep and usually arise from N3. Any disrupttive factor during N3, such as obstructive sleep apnea (OSA), may trigger sleep terrors [5].

Allergic rhinitis affects approximately $40 \%$ of children. Medications used for the treatment of allergies include: corticosteroids, antihistamines, leukotriene modifiers and mast cell stabilizers. Both cetirizine a second-generation oral antihistamine, and montelukast, a selective leukotriene receptor antagonist, minimally cross the blood brain barrier. Prior reports have demonstrated a strong association between montelukast and nightmares. [6]. Insomnia and nocturnal awakenings have been reported with cetirizine. [7].

\section{CONCLUSION}

Night terrors in our patient were attributed to cetirizine due to the resolution of symptoms after discontinuation of the medication and recurrence of symptoms after reintroduction of cetirizine. The mechanism of sleep terrors with cetirizine may be secondary to arousals from N3. Our patient remains symptom free after discontinuation of cetirizine. This case highlights the importance of evaluating for possible medication effect with sudden onset parasomnias.

\section{REFERENCES}

[1] Laberge, L., Tremblay, R.E., Vitaro, F. and Montplaisir, J. (2000) Development of parasomnias from childhood to early adolescence. Pediatrics, 106, 67-74. doi:10.1542/peds.106.1.67

[2] American Academy of Sleep Medicine (2005) International classification of sleep disorders; diagnostic and coding manual. 2nd Edition, American Academy of Sleep Medicine, Westchester.

[3] Nguyen, B.H., Perusse, D., Paquet, J., Petit, D., Bolvin, M., Tremblay, R.E. and Montplaisier, J. (2008) Sleep terrors in children: A prospective study of twins. Pediatrics, 122, e1164-e1167. doi:10.1542/peds.2008-1303

[4] Owens, J.A., Millman, R.P. and Spirito, A. (1999) Sleep terrors in a 5-year-old girl. Archives of Pediatrics \& Adolescent Medicine, 153, 309-312.

[5] Owens, J., Spirito, A., Nobile, C. and Arrigan, M. (1997) Incidence of parasomnias in children with obstructive sleep apnea. Sleep, 20, 1193-1196.

[6] Wallerstedt, S.M., Brunlof, G., Sundstrom, A. and Eriksson, A.L. (2009) Montelukast and psychiatric disorders in children. Pharmacoepidemiology and Drug Safety, 18, 858-864. doi:10.1542/peds.2008-1303

[7] Jones, M.C. and Jones, C.E. Jr. (2010) Use of cetirizine in a 23-month-old male causes insomnia. Southern Medical Journal, 103, 485-486. doi:10.1097/SMJ.0b013e3181b9ae49 